Indonesian Journal of Physics

Vol 18 No. 2 April 2007

\title{
Comparison of Electron Direct Transmittance and Tunneling Time of Si (100)/HfO2/Si (100) and Si (110)/HfO2/Si (110) Structures with Ultra-thin Trapezoidal Barrier
}

\author{
Fatimah A. Noor, ${ }^{1)}$ Mikrajuddin Abdullah, Sukirno, and Khairurrijal ${ }^{2)}$ \\ Physics of Electronic Materials Research Division, \\ Faculty of Mathematics and Natural Sciences, \\ Institut Teknologi Bandung, Bandung \\ 1) e-mail: fatimah@students.itb.ac.id; ${ }^{2)}$ krijal@fi.itb.ac.id
}

\begin{abstract}
An analytical expression of electron direct transmittance and tunneling time through a nanometer-thick trapezoidal potential barrier have been derived by using a phase-time method with Airy wavefunction solution. The expression is applied to $\mathrm{Si}(100) / \mathrm{HFO}_{2} / \mathrm{Si}$ (100) (isotropic) and $\mathrm{Si}(110) / \mathrm{HfO}_{2} / \mathrm{Si}(110)$ (anisotropic) structures calculated under the consideration of barrier width, incident energy, incident angle, and bias voltage. The calculated results are discussed and comparisons between the isotropic and anisotropic heterostructures are discussed.
\end{abstract}

Keywords: mass tensor, phase-time, transmittance, tunneling time.

\section{Introduction}

The tunneling phenomenon passing through a potential barrier has been discussed since 60 years ago and is still of interest to study quantum transport in heterostructures in present day. The concept of tunneling time is very important for ultimate performance evaluations of resonant tunneling diodes, traveling-wave tunnel monolithic integrated circuits, and infrared resonant tunneling lasers. ${ }^{1)}$ Recently, the subject has received considerable attention in view of potential use of these structures in device fabrication. ${ }^{2,3)}$

There have been many studies on the tunneling time and proposed some models to solve the tunneling time. One of the method in calculating the tunneling time is a phase time method which was introduced by Bohm ${ }^{4)}$ and Wigner ${ }^{5)}$ where is the best model of the tunneling time as proved by Steinberg and Chiao ${ }^{6)}$.

Very recently, Khairurrijal et al. ${ }^{7)}$ have adopted the Wigner's phase time approach to calculate the one-dimensional electron tunneling time in an isotropic heterostructure with exponential wavefunction solution. In this paper, we have derived analytical expresssions of electron direct transmittance and tunneling time of $\mathrm{Si}(100) / \mathrm{HfO}_{2} / \mathrm{Si}(100) \quad$ (isotropic) and $\mathrm{Si}(110) / \mathrm{HfO}_{2} / \mathrm{Si}(110)$ (anisotropic) structures by using phase time method with airy wiavefunction solution. The analytical expressions were calculated under the consideration of barrier width, incident energy, incident angle, and bias voltage. The calculated results are discussed and compared between the isotropic and anisotropic heterostructures.

\section{Theoretical model}

Figure 1 shows the schematic energy diagram of a heterostructure with a trapezoidal potential barrier in the normal direction (z-direction) to the layer. The barrier width is $L$ and the barrier height is $\Phi$. The electron effective mass and potential are dependent on the $z$-direction. It is considered that the incident energy $E$ is smaller than the barrier height $\Phi$ and the effective mass of an electron in region I is the same as that in region III $\left(m_{1}\right)$ and in region II that is $m_{2}$.

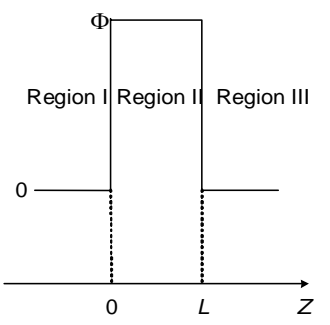

(a)

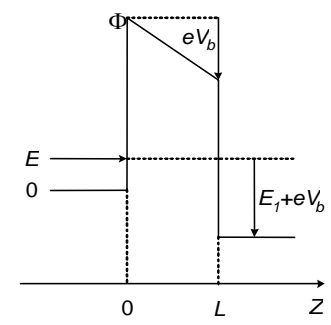

(b)
Figure 1. Energy band profile without giving bias voltage (a) and giving bias voltage to the barrier (b).

The Hamiltonian for the general anisotropic materials is expressed as follow ${ }^{4}$ :

$$
H=\frac{1}{2 m_{0}} \bar{p}^{T} \cdot \alpha(\bar{r}) \cdot \bar{p}+V(\bar{r}),
$$

where $m_{0}$ is the free electron mass, $\bar{p}$ is a momentum vector, $\left(1 / m_{0}\right) \alpha$ is the inverse effective mass tensor which expressed as

$$
\alpha(\mathbf{r})=\left(\begin{array}{lll}
\alpha_{x x} & \alpha_{x y} & \alpha_{x z} \\
\alpha_{y x} & \alpha_{y y} & \alpha_{y z} \\
\alpha_{z x} & \alpha_{z y} & \alpha_{z z}
\end{array}\right),
$$


and $V(\bar{r})$ is the potential energy.

For the isotropic material, the effective invers mass tensor is written as

$$
\alpha(\mathbf{r})=\left(\begin{array}{ccc}
\alpha_{x x} & 0 & 0 \\
0 & \alpha_{y y} & 0 \\
0 & 0 & \alpha_{z z}
\end{array}\right),
$$

where $\alpha_{x x}=\alpha_{y y}=\alpha_{z z}=\alpha$ then $\gamma=0$

The effective-mass equation wave function with the Hamiltonian of Eq. (1) is given by

$$
\Psi(\bar{r})=\varphi(z) \exp (-i \gamma z) \exp \left(i\left(k_{x} x+k_{y} y\right)\right),
$$

where

$$
\gamma=\frac{k_{x} \alpha_{x z}+k_{y} \alpha_{y z}}{\alpha_{z z}} .
$$

$\varphi(z)$ satisfies the one-dimensional Schrödinger-like equation in this problem

$$
-\frac{\hbar^{2}}{2 m_{0}} \alpha_{z z, m} \frac{\partial^{2} \varphi(z)}{d z^{2}}+V(z) \varphi(z)=E_{z} \varphi(z),
$$

where the subscript $\mathrm{m}$ in $\alpha_{z z, m}$ denotes each region in Fig. 1 and the electron energy in the normal direction can be written as

$$
E_{z}=E-\frac{\hbar^{2}}{2 m_{0}} \sum_{i, j \in\{x, y\}} \beta_{i j} k_{i} k_{j},
$$

where

$$
\begin{aligned}
& E=\sum_{i, j \in\{x, y, z\}} \frac{\hbar^{2}}{2 m_{0}} \alpha_{i j, m} k_{i} k_{j}, \\
& \beta_{i j}=\alpha_{i j}-\frac{\alpha_{i z} \alpha_{z j}}{\alpha_{z z}},
\end{aligned}
$$

where $\alpha_{i j}$ is the inverse effective-mass tensor.

We further consider the time-independent electron wave function in each region in Fig. 1 as

$$
\psi(z)=\left\{\begin{array}{lr}
A \exp \left(i k_{1} z\right)+B \exp \left(-i k_{1} z\right), & z \leq 0 \\
C A i(\varsigma(z))+D B i(\varsigma(z)), & 0<z<L \\
G \exp \left(i k_{3} z\right)+H \exp \left(-i k_{3} z\right), & z \geq L
\end{array}\right.
$$

where $A, C, G$ are transmission coefficient in each region, $B, D, H$ are reflection coefficient in each region, and $A i$ and $B i$ are the Airy function.

The wave number $k_{1}$ and $k_{3}$ are expressed respectively as follow as

$$
\begin{aligned}
& k_{1}=\left(\frac{2 m_{1} E}{\hbar^{2}}\right)^{1 / 2}, \\
& k_{3}=\left(\frac{2 m_{1}\left(E+e V_{b}\right)}{\hbar^{2}}\right)^{1 / 2},
\end{aligned}
$$

where $\hbar$ is the reduced Planck constant and $V_{b}$ is the voltage applied to the barrier. The argument $\zeta(z)$ of the Airy functions is expressed as

$$
\varsigma(z)=\left(\frac{2 m F}{\hbar^{2}}\right)^{1 / 3}\left(\frac{\Phi-E}{F}-z\right),
$$

where $F=e V_{b} / L$ is the electric field in the barrier.

With the boundary conditions at $z=0$ and $z=L$, which are given by ${ }^{8}$

$$
\begin{array}{cc}
\psi_{I}\left(z=0^{-}\right)=\psi_{I I}\left(z=0^{+}\right), & \left.\frac{1}{m_{1}} \frac{d \psi_{I}}{d z}\right|_{z=0^{-}}=\left.\frac{1}{m_{2}} \frac{d \psi_{I I}}{d z}\right|_{z=0^{+}}, \\
\psi_{I I}\left(z=L^{-}\right)=\psi_{I I I}\left(z=L^{+}\right), & \left.\frac{1}{m_{2}} \frac{d \psi_{I I}}{d z}\right|_{z=L^{-}}=\left.\frac{1}{m_{1}} \frac{d \psi_{I I I}}{d z}\right|_{z=L^{+}},
\end{array}
$$

the relation between the constants $G$ and $A$ can be written as

$$
\begin{aligned}
f & \equiv \frac{G}{A} \\
& =2 i\left(\frac{k_{1}}{m_{1}} \frac{f_{1}}{\left(\frac{2 e V_{b}}{m_{2} \hbar^{2} L}\right)^{1 / 3} f_{2}+i\left(\frac{k_{1}}{m_{1}} f_{3}+\frac{k_{3}}{m_{1}} f_{4}\right)-\frac{k_{1} k_{3}}{m_{1}{ }^{2}}\left(\frac{2 e V_{b}}{m_{2} \hbar^{2} L}\right)^{-1 / 3} f_{5}}\right) e^{-i k_{3} L}
\end{aligned}
$$

where,

$$
\begin{aligned}
& f_{1}=A i^{\prime}(\varsigma(L)) B i(\varsigma(L))-A i(\varsigma(L)) B i^{\prime}(\varsigma(L)) \\
& f_{2}=A i^{\prime}(\varsigma(L)) B i^{\prime}(\varsigma(0))-A i^{\prime}(\varsigma(0)) B i^{\prime}(\varsigma(L)) \\
& f_{3}=A i(\varsigma(0)) B i^{\prime}(\varsigma(L))-A i^{\prime}(\varsigma(L)) B i(\varsigma(0)) \\
& f_{4}=A i(\varsigma(L)) B i(\varsigma(0))-A i^{\prime}(\varsigma(0)) B i(\varsigma(L)) \\
& f_{5}=A i(\varsigma(0)) B i(\varsigma(L))-A i(\varsigma(L)) B i(\varsigma(0))
\end{aligned}
$$

The transmittance $T_{c}$ of an electron through the trapezoidal barrier is expressed as

$$
T_{c}=f . f^{*} \text {. }
$$

The direct tunneling time $\tau$ is obtained by using the Wigner phase time approach. ${ }^{9}$

$$
\tau=\frac{m_{1}}{\hbar k_{3}}\left(\frac{\partial \phi}{\partial k_{3}}+L\right) \text {. }
$$

\section{Calculated results and discussion}

We use $\mathrm{Si}(100) / \mathrm{HfO}_{2} / \mathrm{Si}(100)$ (isotropic) and $\mathrm{Si}(110) / \mathrm{HfO}_{2} / \mathrm{Si}(110)$ (anisotropic) structures MOS (metal-oxide-semiconductor) structures to examine the theoretical model described in the previous section by applying a bias voltage $V_{b}$ to the barrier. The following parameters are given : $\Phi=1.5 \mathrm{eV}, m_{2}=0.15 m_{0}$, where $m_{0}$ is the free electron mass to calculate the electron transmittance and tunneling time. For anisotropic material, there are four equivalent valleys in the conduction band of $\mathrm{Si}(110)^{10)}$. The effective mass tensor elements of these four valleys are not the same. There are two groups of valley in Si (110), each of them has two valleys. The invers effective mass tensors used in our numerical calculation are shown in table 1. 
Table 1. Inverse effective-mass tensors $\left(\alpha_{i j}\right)$ of $\mathrm{Si}$ (110) used in the numerical calculation.

\begin{tabular}{|c|ccc|}
\hline Valley & \multicolumn{3}{|c|}{ Regions I and III } \\
\hline \multirow{3}{*}{1} & $\left(\begin{array}{ccc}5.26 & 0 & 0 \\
0 & 3.14 & 2.12 \\
0 & 2.12 & 3.14\end{array}\right)$ \\
\hline \multirow{2}{*}{2} & $\left(\begin{array}{ccc}5.26 & 0 & 0 \\
0 & 3.14 & -2.12 \\
0 & -2.12 & 3.14\end{array}\right)$ \\
\hline
\end{tabular}

Figure 2 shows the coordinate system used in our problem. The position where the incident electron hits the barrier is the origin of the coordinate system. From this system, the eq. (6) to be

$$
\begin{aligned}
& E=\frac{\hbar^{2}}{2 m_{0}}\left\{\alpha_{x x, 1} k^{2} \sin ^{2} \theta \cos ^{2} \varphi+\alpha_{y y, 1} k^{2} \sin ^{2} \theta \sin ^{2} \varphi\right. \\
& +\alpha_{z z, 1} k^{2} \cos ^{2} \theta+2\left(\alpha_{x y, 1} k^{2} \sin ^{2} \theta \cos \varphi \sin \varphi\right. \\
& \left.\left.+\alpha_{y z, 1} k^{2} \sin \theta \cos \theta \sin \varphi+\alpha_{z x, 1} k^{2} \sin \theta \cos \theta \cos \varphi\right)\right\}
\end{aligned}
$$

We calculate the direct tunneling time with the angle of incident electron are $\theta$ and $\varphi$ respectively. We fix $\varphi$ to $\pi / 2$ and change only $\theta$ for simplicity.

The calculated results are plotted as a function of barrier width, incident energy, incident angle, and bias voltage as the figures below. The solid line is for an electron in $\mathrm{Si}(100) / \mathrm{HfO}_{2} / \mathrm{Si}(100)$ (isotropic) structures, and and the dotted line is for an electron in $\mathrm{Si}(110) / \mathrm{HfO}_{2} / \mathrm{Si}(110)$ (anisotropic) structures.



Figure 2. The coordinate system used in this paper

Figure 3 and figure 4 show the plot of barrier width dependence of the direct transmittance and tunneling time respectively. Keeping the energy of electron and the bias voltage constant are $0.2 \mathrm{eV}$ and $0.1 \mathrm{~V}$ respectively. The data are plotted for two structures for incident angle is $0^{\circ}$. Both of isotropic and anisotropic structure, it is clearly seen that for $\mathrm{L}<2 \mathrm{~nm}$, the direct transmittance decreases incisively as the $\mathrm{HfO}_{2}$ layer becomes thicker. For $L$ is thicker than $2 \mathrm{~nm}$, the direct transmittance tends to constant, independent of the thickness of $\mathrm{HfO}_{2}$ layer. The transmittance of isotropic structure is higher than anisotripic structure.

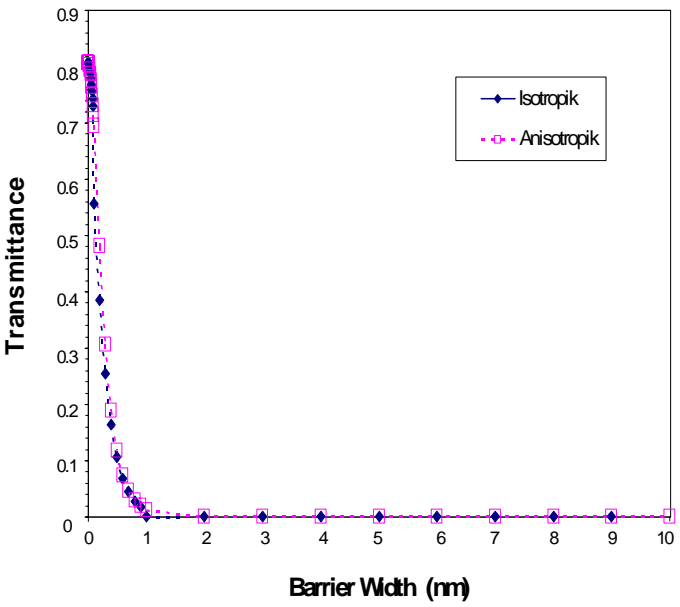

Figure 3. Direct transmittance depends on barrier width with incident electron energy $\mathrm{E}=0.2 \mathrm{eV}$ and bias voltage $\mathrm{V}_{\mathrm{b}}=0.1 \mathrm{~V}$.

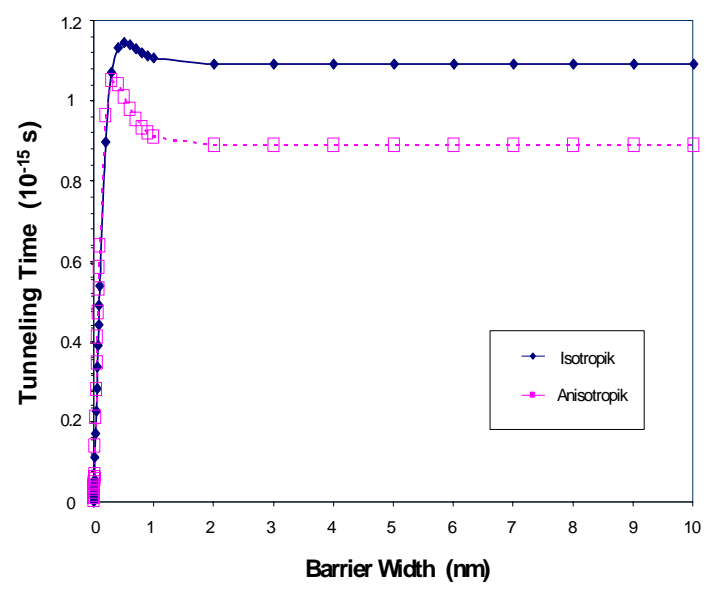

Figure 4. Direct tunneling time depends on barrier width with incident electron energy $\mathrm{E}=0.2 \mathrm{eV}$ and bias voltage $\mathrm{V}_{\mathrm{b}}=0.1 \mathrm{~V}$.

It is clearly seen that for $\mathrm{L}<0.5 \mathrm{~nm}$, the direct tunneling time $\tau$ increases incisively as the $\mathrm{HfO}_{2}$ layer becomes thicker in both of the isotropic and anisotropic structures. For $L$ is thicker than $0.5 \mathrm{~nm}$, the direct tunneling time decreases as the oxide thickness as increases. As . $\mathrm{L}>2 \mathrm{~nm}$ the direct tunneling time tends to constant, independent of the thickness of $\mathrm{HfO}_{2}$ layer. Generally, As the $\mathrm{HfO}_{2}$ layer becomes thicker, the direct tunneling time for isotropic structure is less than anisotropic structure.

Figure 5 and figure 6 respectively, show the incident energy of electron dependence of the direct transmittance and tunneling time with the bias voltages of $0.1 \mathrm{~V}$ and incident angle is $0^{\circ}$. Taking the barrier width $\mathrm{L}=1 \mathrm{~nm}$, the direct transmittance becomes increases as the incident energy becomes increase for both of isotropic and anisotropic structure. It is shown that as incident energy increases, the probability of an electron through the barier in isotropic structur is 
higher than in anisotropic structure. Both of isotropic and anisotropic structure, the direct tunneling time becomes decrease for the incident energy becomes increase. For both isotropic and anisotropic structure, increasing of incident energy is able to accelerate electron passing through the barrier which the movement of electron in isotropic structure is faster than in anisotropic structure.



Figure 5. Energy of electron dependence of direct transmittance with bias voltage $\mathrm{V}_{\mathrm{b}}=0.1 \mathrm{~V}$ and barrier width $\mathrm{L}=1 \mathrm{~nm}$.

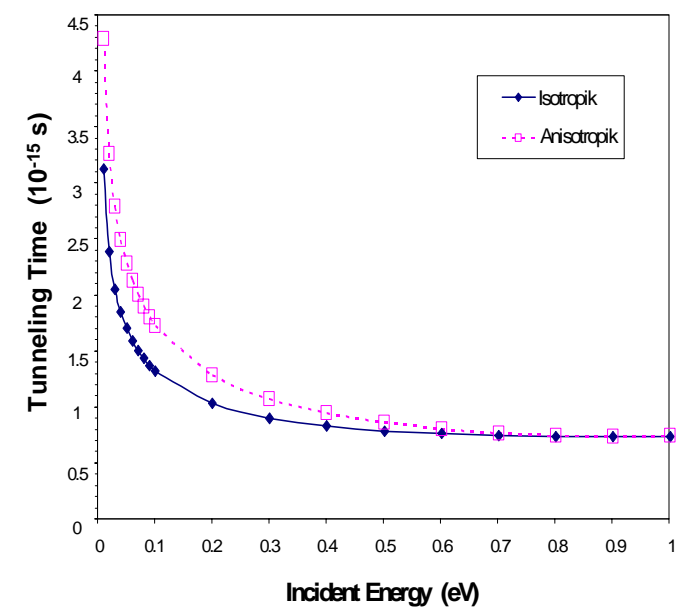

Figure 6. Energy of electron dependence of direct tunneling time with bias voltage $\mathrm{V}_{\mathrm{b}}=0.1 \mathrm{~V}$ and barrier width $\mathrm{L}=1 \mathrm{~nm}$.

Figure 7 and figure 8 respectively show the direct transmittance and tunneling time as a function of varied the angle (in degree) of incidence with respect to the barrier over the range from $-90^{\circ}$ to $90^{\circ}$ with the barrier width $L=1 \mathrm{~nm}$, incident energy of electron $E=0.2 \mathrm{eV}$, and bias voltage $\mathrm{V}_{\mathrm{b}}=0.1 \mathrm{~V}$. It is shown that for both of isotropic and anisotropic structure, the direct transmittance gives the highest value at the incident angle of $0^{\circ}$.
In anisotropic structure for all valleys, the direct transmittance is asymmetric with the change of the incident angle. Both of isotropic and anisotropic structure, the direct tunneling time gives the lowest value at the incident angle of $0^{\circ}$. It is means that for the incident angle of $0^{\circ}$, the movement of electron becomes fastest. In anisotropic structure for all valleys, the direct transmittance is asymmetric with the change of the incident angle.



Incident Angle (degree)

Figure 7. Direct transmittance as function of angle with incident electron energy $\mathrm{E}=0.2 \mathrm{eV}$ and bias voltage $\mathrm{V}_{\mathrm{b}}=0.1 \mathrm{~V}$.

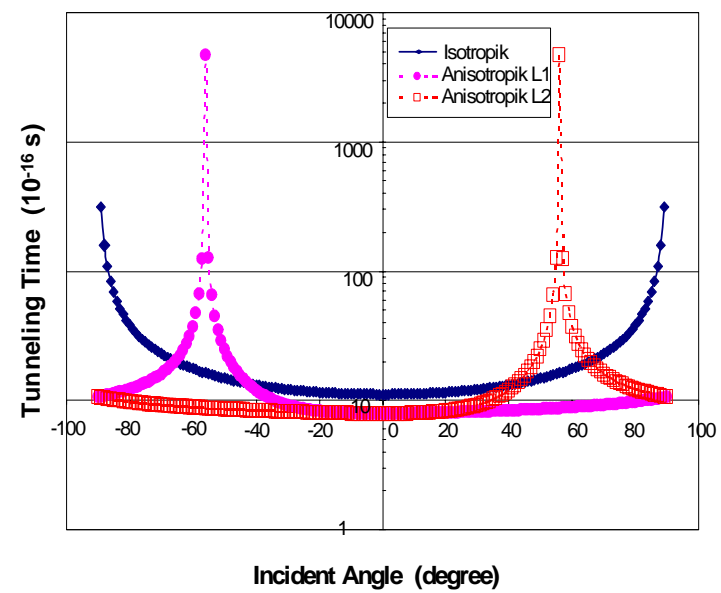

Figure 8. Direct tunneling time as function of angle with incident electron energy $\mathrm{E}=0.2 \mathrm{eV}$ and bias voltage $\mathrm{V}_{\mathrm{b}}=0.1 \mathrm{~V}$.

Figure 9 and figure 10 respectively, show the bias voltage dependence of the direct transmittance and tunneling time with the barrier width $L=1 \mathrm{~nm}$, incident energy of electron $E=0.2 \mathrm{eV}$, and incident angle is $0^{\circ}$. For isotropic structure, the direct transmittance tends to increase for $\mathrm{Vb}<0.3 \mathrm{~V}$. For $\mathrm{Vb}>0.3 \mathrm{~V}$, the direct transmittance tends to decrease exponentially. For anisotropic structure, the direct transmittance tends to be constant, independent of the bias voltage. It is clearly shown that the direct transmittance in isotropic material more higher than anisotropic material. It is also shown, As the bias 
voltage becomes increase, the direct tunneling time for isotropic structure is less than anisotropic structure.

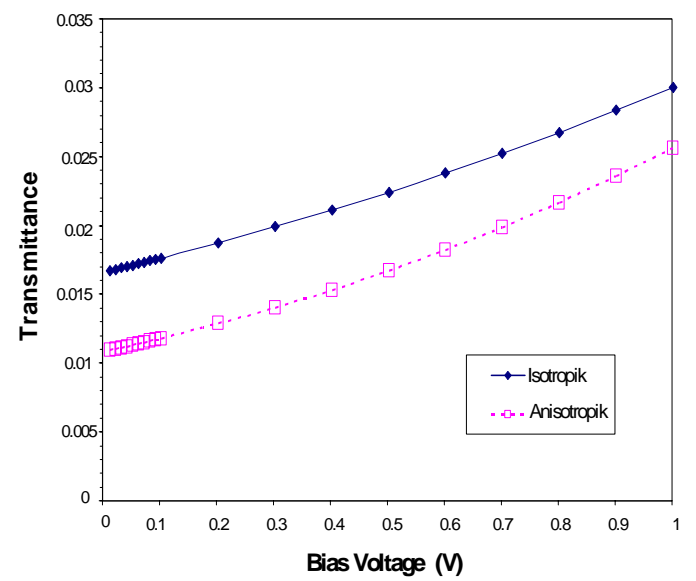

Figure 9. Direct transmittance as function of bias voltage with incident electron energy $\mathrm{E}=0.2 \mathrm{eV}$ and barrier width $\mathrm{L}=1 \mathrm{~nm}$.

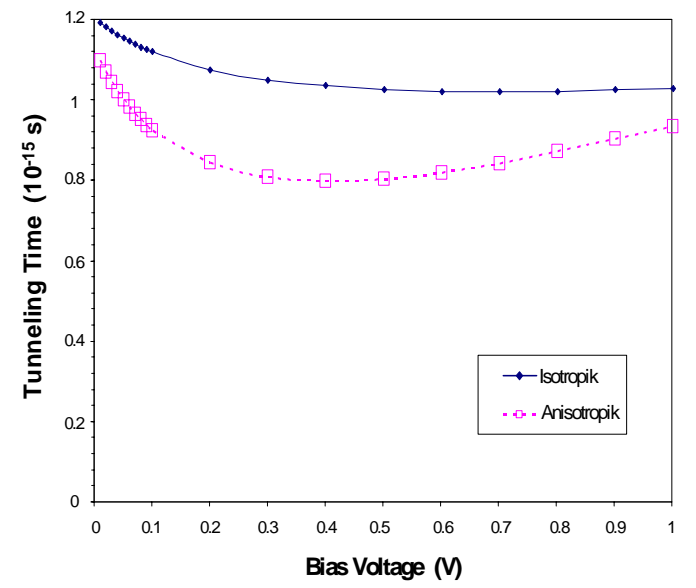

Figure 10. Direct tunneling time as function of bias voltage with incident electron energy $\mathrm{E}=0.2 \mathrm{eV}$ and barrier width $\mathrm{L}=1 \mathrm{~nm}$.

\section{Conclusion}

We have derived an analytical expression of electron direct tunneling time through a nanometerthick trapezoidal barrier isotropic and anisotropic structure. It is found that probability of an electron in isotropic structure through the barrier is higher than in anisotropic structure. It is also found that the electron movement is faster in isotropic structure than in anisotropic structure.

\section{References}

1. D. Dragoman and M. Dragoman, IEEE J Quantum Elect., 32, 1150, 1996.

2. R. Havart, et al., IEEE Trans. Elect. Dev., 45, 2291, 1998.

3. E. Lheurette, et al., IEEE Elect. Dev. Lett., 19, 338, 1998.

4. D. Bohm, Quantum Theory, Prentice Hall, New York, 257, 1951.

5. E. P. Wigner, Phys. Rev., 98, 145, 1955.

6. A. M. Steinberg and R. Y. Chiao, Phys. Rev. A., 51, 3525, 1995.

7. Khairurrijal, F. A. Noor, and Sukirno, Solid-State Electron, 49, 923, 2005.

8. K. -Y. Kim and B. Lee, Phys. Rev. B., 58, 6728, 1998.

9. B. Lee, Superlattices Microst., 14, 295, 1993.

10. C. Lee, Intersubband Absorption in Conduction Bands of Silicon and Germanium Quantum Wells, UCLA, 1994. 